\title{
Effects of fluoxetine on the oral environment of bulimics
}

\author{
W. A. Bretz ${ }^{1}$, D. D. Krahn², M. Drury ${ }^{3}$, \\ N. Schork ${ }^{4}$, W. J. Loesche ${ }^{3,5}$ \\ ${ }^{1}$ Department of Cariology and General \\ Dentistry, School of Dentistry, ${ }^{2}$ Department \\ of Psychiatry, School of Medicine, \\ ${ }^{3}$ Department of Biologic and Materials \\ Sciences, School of Dentistry, ${ }^{4}$ Department of \\ Internal Medicine, School of Medicine, \\ ${ }^{5}$ Department of Microbiology and \\ Immunology, School of Medicine, University of \\ Michigan, Ann Arbor, USA
}

\author{
Bretz WA, Krahn DD, Drury M, Schork N, Loesche WJ. Effects of fluoxetine on \\ the oral environment of bulimics. \\ Oral Microbiol Immunol 1993: 8: 62-64. (C) 1993 Munksgaard
}

\begin{abstract}
The purpose of this study was to evaluate in a double-blind placebo-based study the effects of fluoxetine over a period of 16 weeks on the frequency of binging and purging and on fluctuations in the levels of cariogenic organisms and saliva secretion rate of patients $(n=30)$ with bulima nervosa. Profile analysis suggested that, over the course of the study, binging and purging frequency and Streptococcus sobrinus salivary levels decreased significantly in the fluoxetine group as compared with the placebo group. Our finding that $S$. sobrinus levels decreased 16 weeks after subjects were on medication suggests that the salivary levels of these organisms could serve as an objectively measured indicator of patient compliance with antibulimic therapy.
\end{abstract}

Key words: bulimia; Streptococcus mutans; Streptococcus sobrinus; lactobacilli; yeast; saliva; fluoxetine

W. A. Bretz, Department of Cariology and General Dentistry, School of Dentistry, University of Michigan, Ann Arbor, MI 48109, USA Accepted for publication June 6, 1992
Fluoxetine is a specific blocker of serotonin reuptake (12) that has been used to decrease binge-eating and vomiting in patients with bulimia nervosa $(6-8$, 11). We have previously reported (4) that Streptococcus sobrinus appears to be an indicator of bulimic behavior (active binging and purging). The purpose of the present study was to evaluate in a double-blind placebo-based study the effects of fluoxetine over a period of 16 weeks on the frequency of binging and purging, on fluctuations in the levels of cariogenic organisms and on the rate of saliva secretion of patients with bulimia nervosa.

This investigation was performed on a subset of subjects who participated in a multicenter, double-blind, placebocontrolled study of the efficacy and safety of fluoxetine. Approximately 400 patients at 15 different sites comprised the trial study group. The multicenter protocol included a 1-week, no-therapy screening period and' a 2 -week, singleblind placebo lead-in period. The study subjects at the University of Michigan site comprised 30 patients (29 women and 1 man), 23 randomized to fluoxetine treatment and 7 to placebo treatment. Patients from both groups were well matched on age, age of onset of bulimic behavior, height and weight. The patients received $60 \mathrm{mg}$ orally each day, over a course of 16 weeks. Compliance was assessed by pill counts performed at each visit. Assessment of frequency of binging and purging and of oral microbial and salivary parameters were performed at the end of the 2week, single-blind placebo period (baseline); after 8 weeks on active drug or placebo; and after 16 weeks on active drug or placebo.

In order to be included in the study, patients must have reported binge-eating and vomiting at least 3 times per week for at least 6 months and had to meet all DSM-III-R (3) criteria for bulimia nervosa. Symptoms must not have decreased markedly during the placebo lead-in period. Binging and vomiting frequencies were assessed by a patient diary that was collected at each visit. We developed a scale in order to rate bingepurge severity: $1:<$ than once a week; 2 : once a week; 3 : twice a week; $4: 3$ times a week; $5: 4$ to 6 times a week; $6: \geq 7$ times a week. This scale was developed to reflect changes in behavior that might be particularly relevant to changes in the oral flora of the patients.

Paraffin-stimulated whole saliva was collected from subjects for $5 \mathrm{~min}$ from which the saliva secretion rate was determined $(\mathrm{ml} / \mathrm{min})$. The levels of cariogenic organisms were assessed as previously described (4). Briefly, $1 \mathrm{ml}$ of saliva was serially diluted at the ratios of 1:10, 1:100 and 1:1000 in reduced transport fluid and plated on selective media by means of a spiral plating system. TYCSB medium for Streptococcus mutans and S. sobrinus (15), LBS agar for lactobacilli (13), and Saboraud dextrose agar (SAB) for yeasts were employed as selective media for these organisms. The number of colony-forming units (CFU)/ml of saliva was calculated after 2 to 3 days of anaerobic incubation (TYCSB and LBS) and aerobic incubation (SAB). S. mutans and $S$. sobrinus were distinguished by colony morphology. Representative colonies of these organisms from each plate were submitted to a biochemical scheme for the identification of the mutans streptococci proposed by Shklair \& Keene (14). Organisms growing on the TYCSB medium other than S. mutans and $S$. sobrinus were found in $14 \%$ of the plates (10 of 74).

The effects of fluoxetine or placebo on microbial and salivary parameters after 8 weeks and 16 weeks on medication were evaluated (Table 1). None of the microbial parameters differed significantly between fluoxetine and placebo groups. This may have been due to patient attrition resulting in a small sample size or to the small size of the placebo group as dictated by the study designers. Another possible explanation 
Table 1. Effects of fluoxetine or placebo on microbial and salivary parameters after 8 weeks and 16 weeks on medication

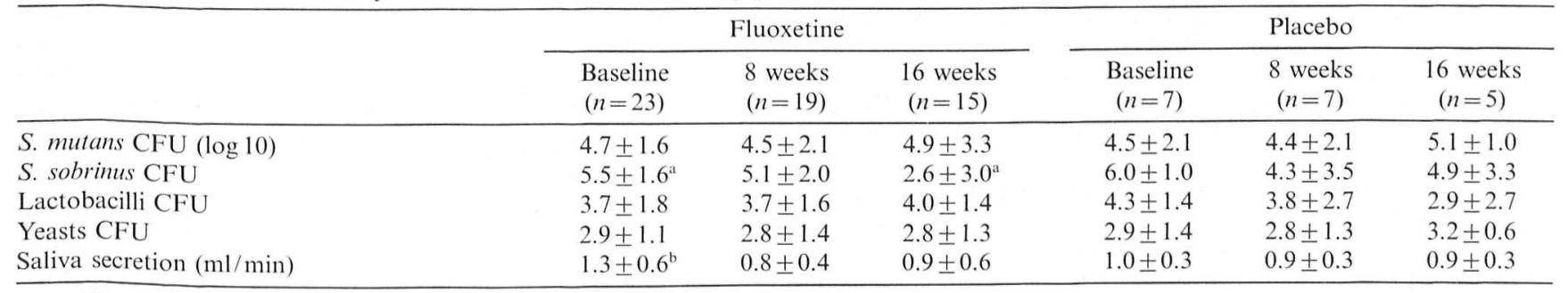

${ }^{a}$ Significantly different by rank paired $t$-test $(P=0.04)$.

b Significantly different by rank paired $t$-test from baseline to 8 weeks $(P<0.0001)$ and from baseline to 16 weeks $(P=0.057)$, in the fluoxetine group.

is related to the reported frequency of binging and purging. This study has shown that subjects from both groups were to a certain extent still binging and purging at the end of the study period, which could contribute to the persistence of aciduric organisms such as the mutans streptococci, lactobacilli and yeasts. The finding of no significant differences between groups in terms of the levels of aciduric organisms may therefore reflect that the selection pressure for aciduric organisms had not been eliminated by fluoxetine intake. The salivary levels of $S$. sobrinus, however, significantly decreased at the 16-week visit in the fluoxetine group (Table 1).

Within the fluoxetine group, the saliva secretion rate was significantly decreased after 8 weeks, a finding that seems to have persisted into the 16th week of the study (Table 1). The fact that the saliva secretion rate measured objectively significantly decreased in the subjects receiving fluoxetine is an interesting finding, as virtually no studies have assessed the rate of saliva secretion objectively before and after the use of antidepressants. Parotid enlargement with dysfunction has been observed

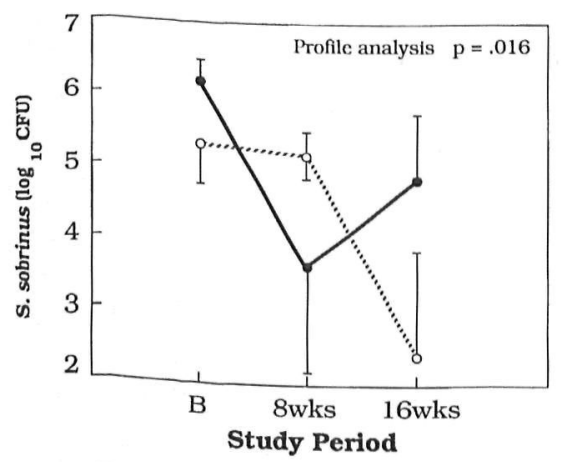

Fig. 1. Effects of fluoxetine on salivary levels of $S$. sobrinus, B: baseline. $O$ : fluoxetine group $(n=15)$; mean \pm SE. $\bullet$ : placebo group $(n=5)$; mean $\pm \mathrm{SE}$. among bulimics due to vomiting $(1,10)$. Certain conditions are associated with parotid enlargement, such as work hypertrophy and electrolyte imbalance, which may result in decreased salivary function. Indeed, correlations between parotid enlargement and xerostomia were observed in bulimics (2). Thus, the objective measurement of a decrease in saliva secretion rate over the course of this study in the treatment group could be a result of fluoxetine usage, a result of parotid dysfunction or a combination of both.

The data from subjects who completed all phases of the study were analyzed by repeated-measures analysis of variance (profile analysis). Fifteen subjects in the fluoxetine group and 5 subjects in the placebo group had complete data. Profile analysis indicated that the average category frequency of binging $(P=0.0007)$ and purging $(P=0.0009)$ decreased over the course of this study in the fluoxetine group compared with the placebo group, i.e., from an average of at least daily binge-purge episodes to an average of twice a week (data not shown). These results are supported by the combined results from all of the investigative sites of the fluoxetine multicenter study (unpublished data), which showed that, after 16 weeks, fluoxetine compared with placebo significantly reduced the average category frequency of binging and purging ( $>50 \%$ decrease in vomiting or binge-eating episodes).

Profile analysis showed a decrease in $S$. sobrinus salivary levels $(P=0.016)$ over the course of the study in the fluoxetine group compared with the placebo group (Fig. 1), regardless of the small number of subjects in each group. A possible explanation for the decrease of $S$. sobrinus in the fluoxetine group is that $S$. sobrinus is more dependent than $S$. mutans for sucrose-mediated attachment to the tooth enamel pellicle (10).
Accordingly, a decrease in the frequency of sugar ingestion (decrease in the frequency of binging) would not favor its colonization. It was recently demonstrated that $S$. sobrinus is capable of producing acid at low $\mathrm{pH}$ values, whereas other mutans streptococci species ceased acid production at similar $\mathrm{pH}$ values (5). From these studies, we may postulate that $S$. sobrinus may be highly active in acidic environments reflecting current emetic activity, as in the case of bulimic subjects, and that after bulimic episodes (purging) are reduced, a decrease in the levels of $S$. sobrinus may occur.

Our finding that $S$. sobrinus salivary levels were decreased 16 weeks after subjects were on medication suggests that the salivary levels of these organisms could serve as an objectively measured indicator of patient compliance with antibulimic therapy. This is in agreement with our previous observation that, in untreated bulimic individuals, the salivary levels of $S$. sobrinus were elevated relative to a healthy matched control group (4). The finding that $S$. sobrinus could be used to monitor bulimic behavior needs to be reproduced in larger population-based studies. It would be of interest to conduct future studies with the use of more accurate assays for the enumeration of S. sobrinus such as monoclonal antibodies and DNA probes.

\section{References}

1. Abrams RA, Ruff JC. Oral signs and symptoms in the diagnosis of bulimia. $\mathbf{J}$ Am Dent Assoc 1986: 113: 761-764.

2. Altshuler BD, Dechow PC, Waller DA, Hardy BW. An investigation of the oral pathologies occurring in bulimia nervosa. Int J Eating Disord 1990: 9: 191199. 
3. American Psychiatric Association. Diagnostic and statistical manual of mental disorders. 3rd edn., revised. Washington, DC: APA, 1987.

4. Bretz WA, Krahn DD, Drewnowski A, Loesche WJ. Salivary levels of putative cariogenic organisms in patients with eating disorders. Oral Microbiol Immunol 1989: 4: 230-232.

5. de Soet JJ, Toors FA, de Graaff J. Acidogenesis by oral streptococci at different pH values. Caries Res 1989: 23: 14-17.

6. Enas GG, Pope HG, Levine LR. Fluoxetine in bulimia nervosa: double-blind study. In: New Research Program and Abstracts, 142nd Annual Meeting of the American Psychiatric Association. Washington, DC: APA, 1989.

7. Freeman CP, Hampson M. Fluoxetine as a treatment for bulimia nervosa. Int $\mathbf{J}$ Obes 1987: 11: 171-177.

8. Freeman CP, Morris SE, Chesiree KE.
A double-blind controlled trial of fluoxetine versus placebo for bulimia nervosa. In: Proceedings summary, Second International Conference on Eating Disorders. New York: Montefiore Medical Center and International Journal of Eating Disorders, 1988.

9. Harrison JL, George LA, Chetham JL. Therapies for a reduction in dental destruction resulting from the manifestations of bulimia nervosa. Texas Dent J 1984: 101: 11-15.

10. Loesche WJ. Role of Streptococcus mutans in human dental decay. Microbiol Rev 1986: 50: 353-380.

11. Marcus MD, Wing RR, Ewing L, Kern E, McDermott M, Gooding W. A double-blind, placebo-controlled trial of fluoxetine plus behavior modification in the treatment of obese binge-eaters and non-binge eaters. Am J Psychiatry 1990: 47: 876-881.
12. Richelson E, Pfenning M. Blockade by antidepressants and related compounds of biogenic amine uptake into rat brain synaptosomes: most antidepressants selectively block norepinephrine. Eur J Pharmacol 1989: 104: 277-286.

13. Rogosa M, Mitchel JA, Wiseman RF. A selective medium for the isolation and enumeration of oral and fecal lactobacilli. J Bacteriol 1951: 62: 132-137.

14. Shklair IL, Keene HJ. A biochemical scheme for the separation of five varieties of Streptococcus mutans. Arch Oral Biol 1974: 19: 1079-1081.

15. Van Palenstein-Helderman WH, Ijsseldijk M, Huis I'nt veld JH. A selective medium for the two major subgroups of the bacterium Streptococcus mutans isolated from human dental plaque and saliva. Arch Oral Biol 1983: 28: 599-603. 
This document is a scanned copy of a printed document. No warranty is given about the accuracy of the copy. Users should refer to the original published version of the material. 